Fast step-response settling of micro electrostatic actuators operated at low air pressure using input shaping

This article has been downloaded from IOPscience. Please scroll down to see the full text article.

2009 J. Micromech. Microeng. 19074020

(http://iopscience.iop.org/0960-1317/19/7/074020)

View the table of contents for this issue, or go to the journal homepage for more

Download details:

IP Address: 131.180.130.109

The article was downloaded on 08/08/2011 at 10:06

Please note that terms and conditions apply. 


\title{
Fast step-response settling of micro electrostatic actuators operated at low air pressure using input shaping
}

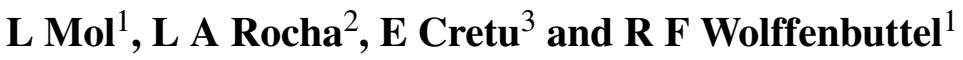 \\ ${ }^{1}$ Faculty EEMCS, Department MicroElectronics/EI, Delft University of Technology, Mekelweg 4, 2828 \\ CD Delft, The Netherlands \\ ${ }^{2}$ Department of Industrial Electronics, University of Minho, Campus de Azurém, 4800-058 Guimarães, \\ Portugal \\ ${ }^{3}$ Department Electrical and Computer Engineering, University of British Columbia, 2332 Main Mall, \\ Vancouver, BC V6T 1Z4, Canada \\ E-mail:1.mol@tudelft.nl
}

Received 21 December 2008, in final form 19 April 2009

Published 30 June 2009

Online at stacks.iop.org/JMM/19/074020

\begin{abstract}
Squeeze-film damping is highly inadequate in low-pressure systems or in systems where air pressure and/or gap dimensions are poorly defined. Input shaping has been used to circumvent the oscillations typically associated with under-damped mass-spring-damper systems and drastically decrease the settling time. The proposed method does not rely on feedback but solely on the system dynamics. The required input signal is derived analytically from the differential equation describing the system. The resulting device response is simulated and experimentally verified on an electrostatically actuated microstructure. Settling occurs even faster than for an equivalent critically damped system.
\end{abstract}

(Some figures in this article are in colour only in the electronic version)

\section{Introduction}

Micro electro-mechanical systems (MEMS) that are used in positioning applications (e.g., micro-mirrors [1], tunable capacitors), desirably have a fast and accurate response to the applied input signal. For MEMS that can be modelled as second-order systems this translates to a critically damped system response. The conventional approach is based on a design in which gas damping in between the moving plate and the static plate (the backplane) is used to obtain a critically damped system.

Low-pressure devices find application in optical MEMS intended for specific applications, where the absorption of light in air has to be avoided. Damping at low pressures close to vacuum has been extensively investigated for high- $Q$ resonator design [2-5]. Although other damping mechanisms have been identified, the main damping mechanism remains gas damping. However in this regime damping should be considered an interaction between free molecules rather than a viscous effect and is much less prominent $[6,7]$. For low- pressure-packaged devices gas damping is typically reduced by two orders of magnitude and gas damping is insufficient. The insufficient damping shows as oscillations (ringing) in the step response, hence, in a much longer setting time.

Eddy-current-based damping could be adopted for providing sufficient damping to ensure a critically damped system. However, the need for a permanent magnetic field arises. This undermines the case for micrometer-sized devices, as permanent magnets are typically bulky and relatively expensive.

Another alternative is to use electronic damping. In principle, any signal that provides a counteracting force proportional to the velocity of the moving object (plate) can be used to provide damping. In the most-straightforward implementation a velocity sensor could be used to generate a voltage that is used in feedback to drive the same structure. Obviously, this would yield a cumbersome system, which is prone to instability. Additionally it can only be applied to differential structures, since the electrostatic damping force is always an attracting force. 


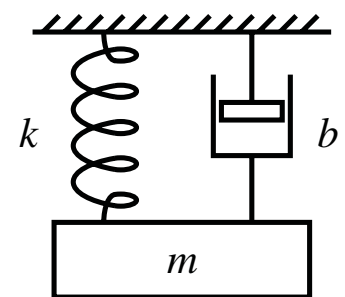

Figure 1. Schematic representation of a MEMS as a mass spring damper system.

This work demonstrates the technique of input shaping [8]. A simple modification (shaping) of the input signal is shown to avoid the oscillations that normally occur when the device settles from a step input. As a consequence the system reaches its steady-state position faster. Input shaping does not rely on feedback, but solely on prior knowledge of the system dynamics. The required input signal is derived analytically from the differential equation describing the system. The approach can be extended to applications where damping is critical and either the dimensions of the moving structure and the air gap or the gas pressure are poorly defined.

In section 2 the technique and the analytical background of input shaping are introduced. Section 3 introduces the device and measurement set-up used for experimental validation. The measurement results are presented in section 4 , followed by the conclusions in section 5.

\section{Input shaping}

The technique of input shaping is well known in the macro world, where large relatively slow systems can benefit greatly from faster settling (e.g., container cranes). It is based on prior knowledge of the system dynamics. By using the prior knowledge, a system can be driven with an input that cancels the unwanted spurious parts of its response.

\subsection{Second-order systems}

MEMS can often be described in a simplified form as the mass spring damper system as shown in figure 1 . Such a system can mathematically be described as a second-order system with differential equation:

$$
m \ddot{x}(t)+b \dot{x}(t)+k x(t)=0,
$$

where $m$ is the mass, $k$ is the spring constant and $b$ is the damping coefficient. Using the following,

$$
\zeta=\frac{b}{2 \sqrt{k m}} \quad \text { and } \quad \omega_{0}=\sqrt{\frac{k}{m}}
$$

(1) can be rewritten in a more generic way as

$$
\ddot{x}+2 \zeta \omega_{0} \dot{x}+\omega_{0}^{2} x=0,
$$

where $\zeta$ is the damping ratio and $\omega_{0}$ is the natural frequency.

The step response of such a system in the case of underdamping $(0 \leqslant \zeta<1)$ is

$$
\begin{aligned}
& 1-\mathrm{e}^{\left(-\zeta \omega_{0} t\right)}\left(\cos \left(\omega_{d} t\right)+\frac{\zeta \sin \left(\omega_{d} t\right)}{\sqrt{1-\zeta^{2}}}\right), \\
& \omega_{d}=\omega_{0} \sqrt{1-\zeta^{2}} .
\end{aligned}
$$

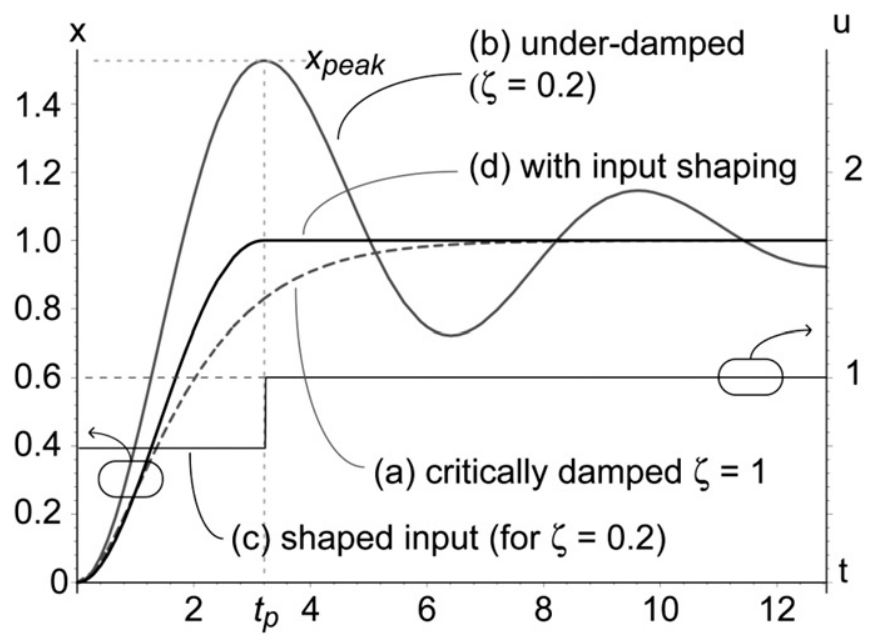

Figure 2. Simulation of the step response of a critically damped (a) and an under-damped $(b)$ system. Also shown is the two-step input waveform $(c)$ for $\zeta=0.2$ that results in a settling-time optimized device response $(d)$.

The time and amplitude of the first overshoot peak can be found by equating the derivative of the step response to zero as (see figure 2)

$$
t_{p}=\pi / \omega_{d} \quad \text { and } \quad x_{\text {peak }}=1+\mathrm{e}^{\left(-\frac{\zeta \pi}{\sqrt{1-\zeta^{2}}}\right)} .
$$

Inspection of the step response unveils the potential for input shaping. Note that at time $t_{p}$ the device has zero velocity. This implies that the device would in principle maintain this position if the input at that moment would correspond to a steady state solution $x_{\text {peak }}$. Expressed differently: for each device position there exists (i) a step input resulting in an overshoot to that position and (ii) a step input with that position as its steadystate solution.

For an initial duration $t_{p}$ the amplitude of the step input is chosen such that its overshoot $x_{\text {peak }}$ corresponds to the desired final position. At time $t_{p}$ the input level is changed to keep the device at its final value set point. Figure 3 illustrates the concept.

\subsection{Input shaping function}

The above time domain analysis shows the concept of input shaping in an intuitive way. A more generalized approach uses the Laplace domain. The second-order system considered here is assumed linear and time invariant. As shown in figure 2, a second step input with adequate amplitude and time exactly cancels the unwanted oscillations. This leads to the concept of an input shaping function. It merely consists of two Dirac pulses:

$$
\text { is }(t)=\frac{1}{x_{\text {peak }}} \delta(t)+\frac{x_{\text {peak }}-1}{x_{\text {peak }}} \delta\left(t-t_{\text {over }}\right) .
$$

Any input signal that is convoluted with the input shaping function is $(t)$ leads to a system response without overshoot. The convolution simplifies to a multiplication in the Laplace domain. The process is shown graphically in figure 4 . 


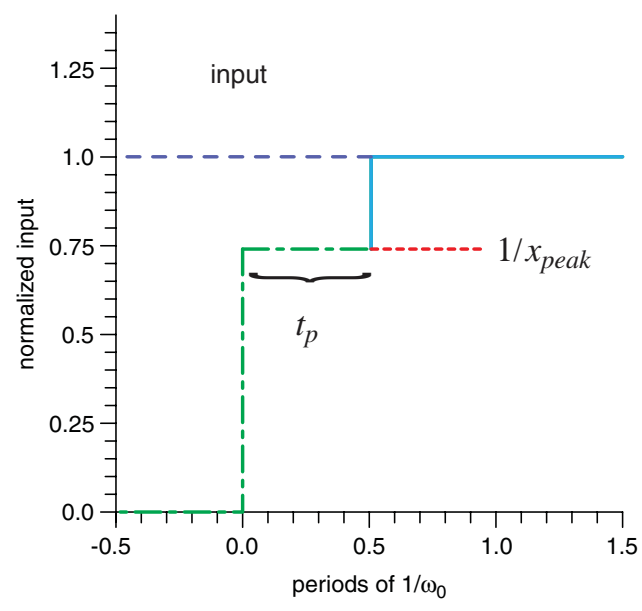

(a)

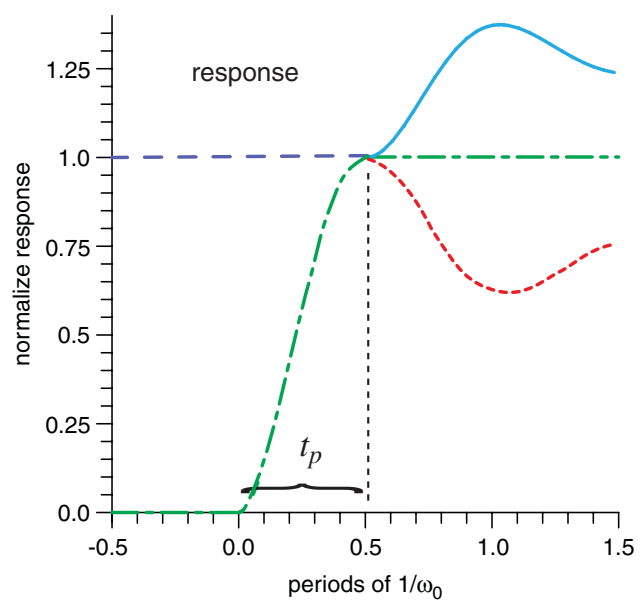

(b)

Figure 3. (a) Input with shaping applied. (b) Response to signal in (a) where the coloured/dashed sections of the line correspond to each other. The green dash-dotted line corresponds to the correct steady-state response. The blue solid line cancels the transient that would appear if the input signal were to continue with the red dotted line.

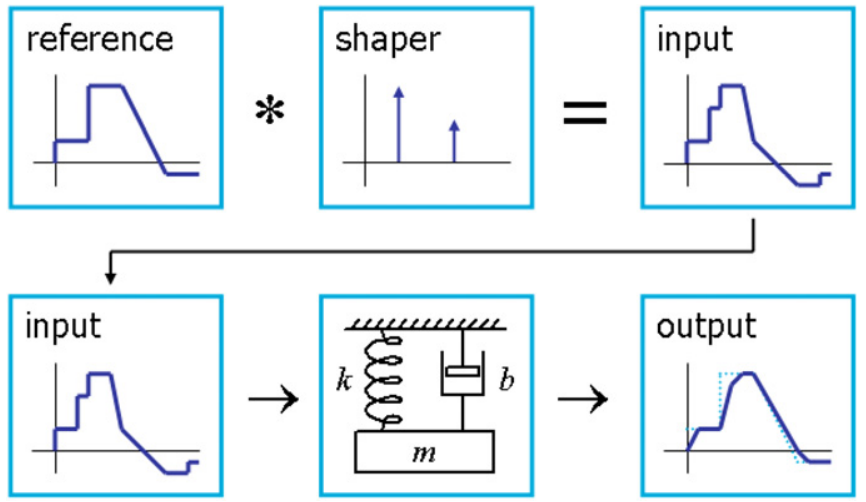

Figure 4. Generalized input shaping example showing the convolution of a reference signal with a shaper consisting of just two Dirac pulses. The shaped input leads to a system response following the reference as close as possible without any overshoot.

\section{Experimental verification}

In order to experimentally verify the concept of input shaping a parallel-plate capacitive MEMS was used. The effectiveness of input shaping is investigated and compared to the expected results from simulation.

\subsection{The electrostatic actuator}

An electrostatically actuated 1-DOF MEMS structure with separate drive and sense electrodes has been designed and fabricated in an SOI process [9] and consists of a central mass suspended on four folded springs (Figure 5). Four distinct sets of electrodes are attached to a central moving bar with counter electrodes at the substrate. The two narrow beam sets located towards the centre of the device form a differential capacitor that is used to read out the device displacement, while each of the wider beam sets is used to excite the mass through electrostatic actuation in either direction. Table 1 lists the key properties of the device.

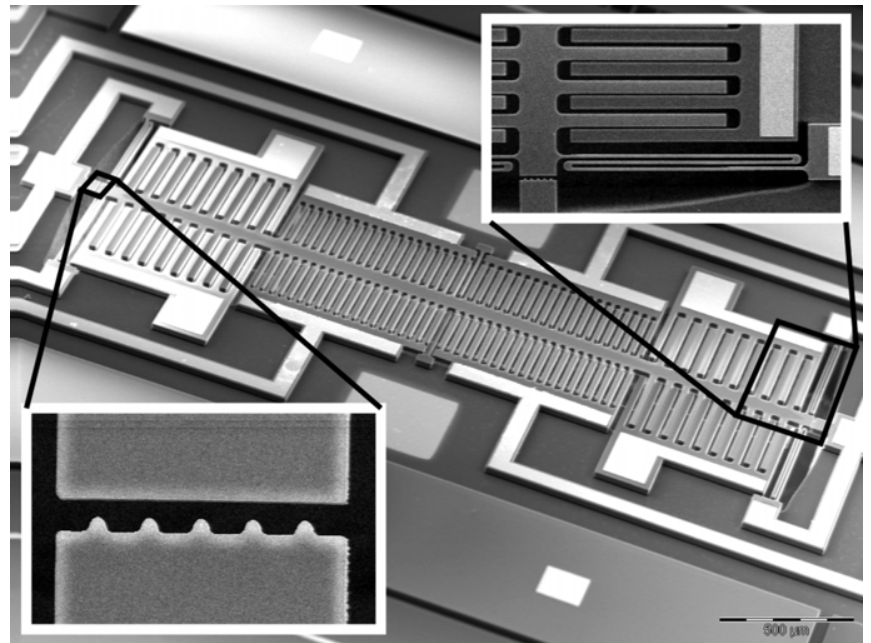

Figure 5. SEM image of the MEMS with the narrow electrodes in the centre used as sensing capacitors and the wider ones on the outside for electrostatic actuation. Insets show details of suspension and stopper.

Table 1. 1-DOF MEMS device properties

\begin{tabular}{lll}
\hline Property & Value & Unit \\
\hline Mass $(m)$ & 42.0 & $\mu \mathrm{g}$ \\
Spring constant $(k)$ & 12.9 & $\mathrm{~N} \mathrm{~m}^{-1}$ \\
Undamped natural frequency & 2.84 & $\mathrm{KHz}$ \\
Sense capacity $\left(C_{s}\right)$ & 1.12 & $\mathrm{PF}$ \\
Device layer thickness $(H)$ & 25.0 & $\mu \mathrm{m}$ \\
Length sense arm $\left(L_{s}\right)$ & 230 & $\mu \mathrm{m}$ \\
Length actuation arm $\left(L_{a}\right)$ & 300 & $\mu \mathrm{m}$ \\
Number of sense arms $\left(N_{\mathrm{sa}}\right)$ & 40 & \\
Number of actuation arms $\left(N_{\mathrm{aa}}\right)$ & 18 & \\
Small gap size $\left(w_{0}\right)$ & 2.29 & $\mu \mathrm{m}$ \\
Large gap size sense electrodes $\left(w_{1 \mathrm{~s}}\right)$ & 14.3 & $\mu \mathrm{m}$ \\
Large gap size actuation electrodes $\left(w_{1 \mathrm{~s}}\right)$ & 12.3 & $\mu \mathrm{m}$ \\
\hline
\end{tabular}

The displacement readout is implemented with a twochannel differential charge amplifier circuit and a coherent detection mixer (figure 6). A $1 \mathrm{MHz}$ carrier signal is injected on the central movable part of the MEMS. Any motion of the 


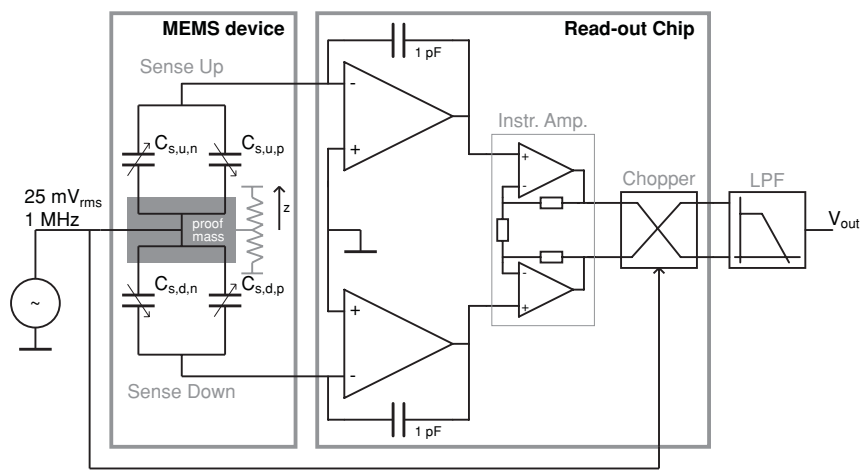

Figure 6. Simplified schematic of the read-out circuit.

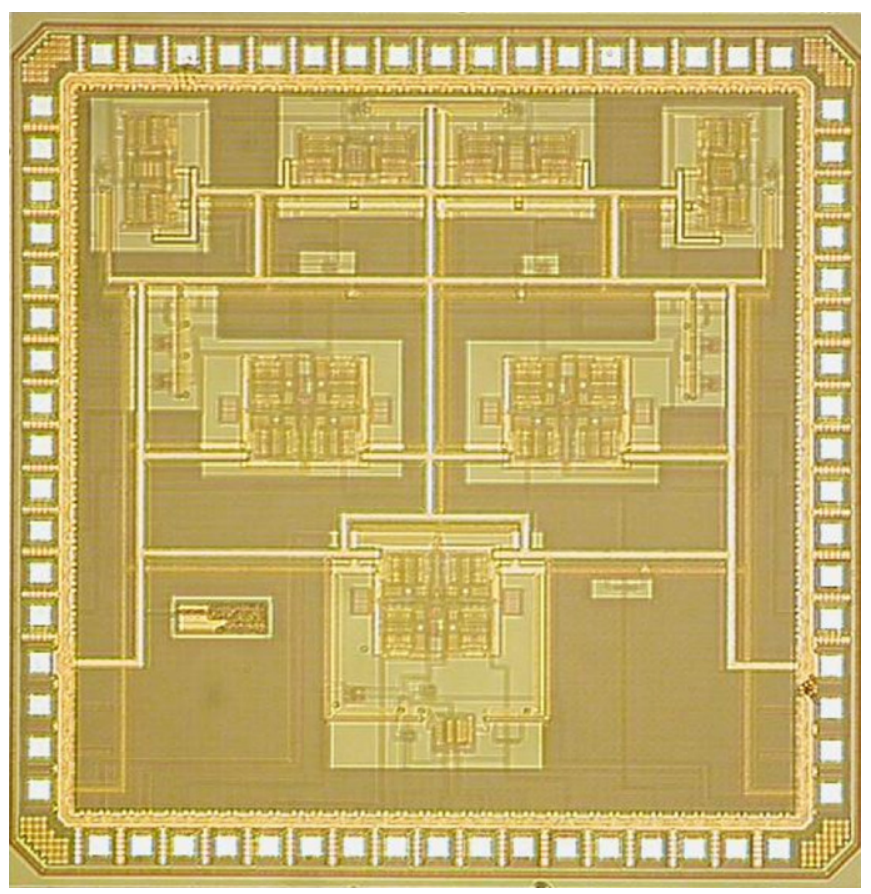

Figure 7. Chip photo of the AMIS $0.35 \mu \mathrm{m}$ CMOS realization of the two-channel differential charge amplifier with a coherent detector circuit.

structure results in an input current of the read-out circuit. The amplified AM modulated carrier signal is coherently detected using a chopper mixer. The readout circuit has been integrated in the AMIS $0.35 \mu \mathrm{m}$ CMOS process and the photograph of the $2.8 \times 2.8 \mathrm{~mm}^{2}$ chip is shown in figure 7 . The overall displacement uncertainty of the combined sensor and readout at a $2.8 \mathrm{kHz}$ bandwidth is $1.6 \mathrm{~nm}$.

\subsection{Pressure-dependent damping}

The amount of squeezed film damping is highly dependent on the pressure of the gas surrounding the structure. By operating the structure inside a vacuum chamber with variable pressure the damping ratio of the resulting second-order system can be controlled. Figure 8 shows a plot of the calculated damping coefficient as a function of pressure. The resulting system is highly under-damped $(\zeta \ll 1)$ for pressures below ambient. The experimental verification was done at a

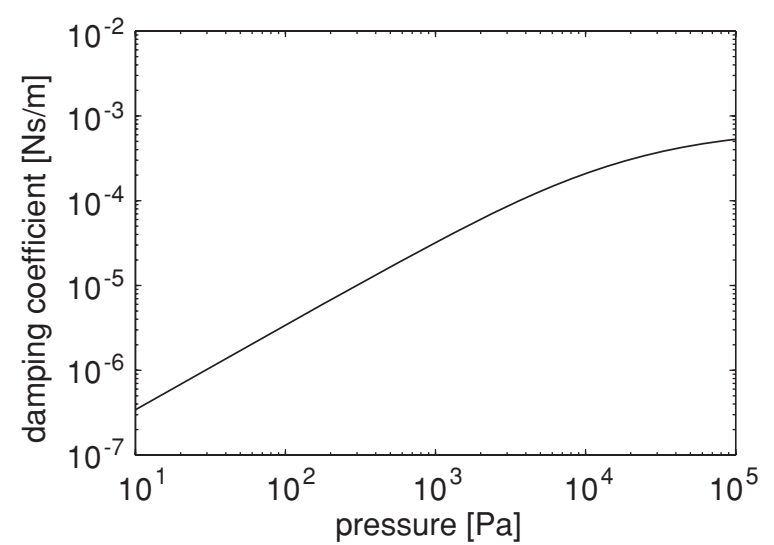

Figure 8. Calculated pressure-dependent damping coefficient for the device under test.

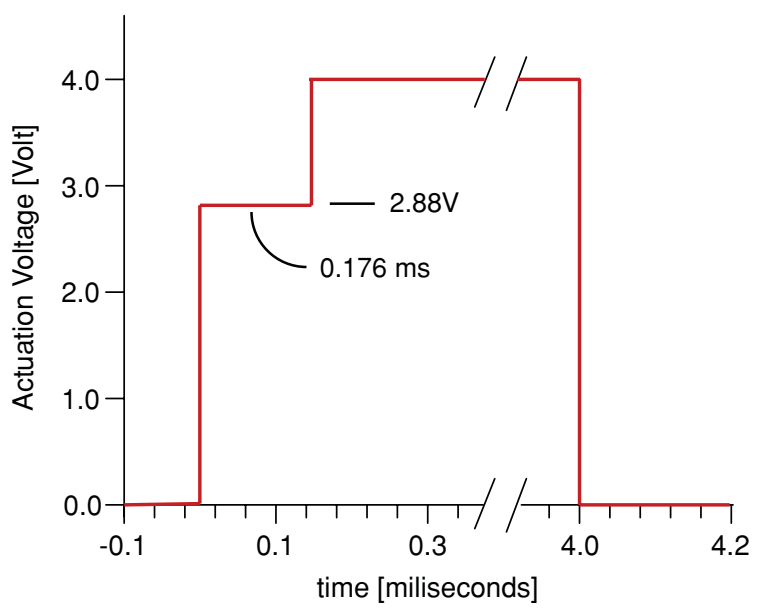

Figure 9. Calculated input shaped electrostatic actuation voltage needed to drive the MEMS at 10 mbar to $22 \%$ displacement of the initial gap, without overshoot. The down-step has not been input shaped for comparison.

pressure of 10 mbar, corresponding to a calculated damping coefficient of $4.5 \times 10^{-5} \mathrm{~N} \mathrm{~s} \mathrm{~m}^{-1}$.

\subsection{Calculated shaped input}

At the chosen operating pressure of 10 mbar, the system has a damping ratio $\zeta$ of 0.0031 . Together with the natural frequency $\omega_{0}$ (table 1), it is used in equations (5) and (6) to determine the input shaping function. In order to verify the concept we calculated the needed shaped input for a device displacement of $22 \%$ of the initial gap size. The resulting two-level step input is defined in terms of force $(\mathrm{N})$, however force cannot be directly set. The actual input variable is the actuation voltage, which generates the electrostatic force and has a quadratic dependence on the applied voltage. The input shaped actuation voltage waveform corrects for this quadratic relation. The waveform is thereafter programmed into a function generator. The removal of the actuation voltage results in a step down to the initial position. In order to compare the difference in settling time, no input shaping is applied to the step back to zero. Figure 9 shows the calculated actuation voltage needed for smooth device movement at 10 mbar. 


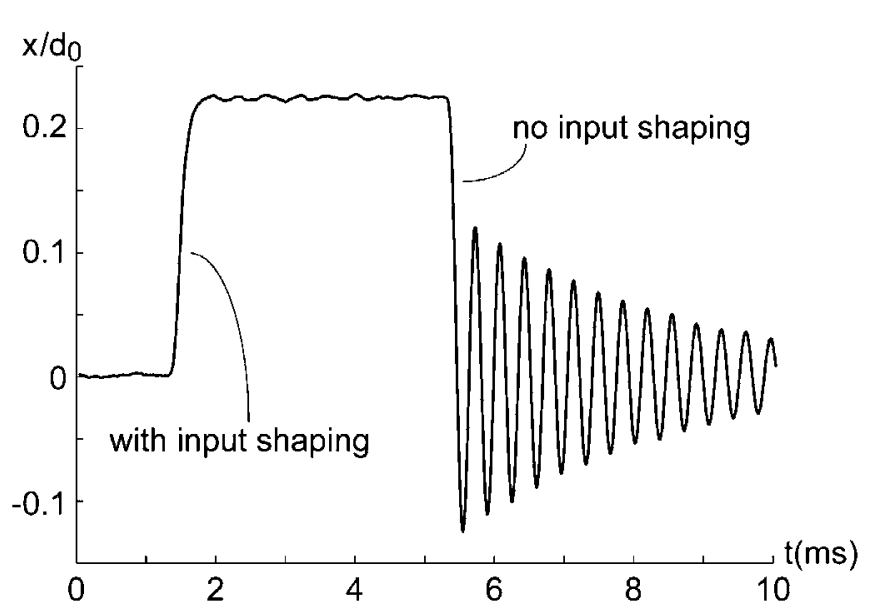

Figure 10. Measured actuator displacement with input shaping applied on the rising edge, however not on the falling edge.

\section{The measurement results}

The structure was packaged in a non-sealed LCC28 carrier and together with the CMOS readout chip mounted on a small PCB. The complete PCB is mounted inside a vacuum chamber with variable pressure, which is regulated to 10 mbar. Vacuum compatible feed throughs allow the passage of electrical signals in and out of the chamber. The structure is actuated with the input shaped voltage step shown in figure 9. The resulting displacement signal was fed to a data acquisition board and sampled at $100 \mathrm{ksamples} / \mathrm{s}$ and further processed in Matlab. The difference in the response is evident from Figure 10. It is interesting to note that the exponential rate of decay of the oscillations contains information on the damping caused by the surrounding gas. With the use of signal processing a curve fit is done on the decay envelope. This confirms the damping coefficient $b$ to be $4.53 \times 10^{-5} \mathrm{~N} \mathrm{~s}$ $\mathrm{m}^{-1}$ at 10 mbar as calculated and also confirms the simulated results in figures 2 and 3.

\section{Conclusions and future work}

Input shaping for obtaining damping without squeeze film air damping has been demonstrated. The technique is relatively simple and can be applied without feedback. However, the technique does depend on prior knowledge on the dynamics of the structure and the gas pressure. In order to determine the shaping function the natural frequency and damping ratio need to be known. The technique is robust in the sense that deviations from the optimal waveform due to uncertainties in device parameters merely result in a slightly longer settling time.

Although the reduction of settling time depends on the damping ratio of the system and the selected settling threshold, it can easily be orders of magnitude lower compared to step inputs without input shaping.

The most interesting applications are in driving an array of MEMS (e.g., micro-mirrors). Within this framework further analysis of the technique is required on two issues. First, the compatibility with a row/column drive scheme with a common readout. Second, the susceptibility of the technique to variations in the structural dimensions and uncertainties in the gas pressure.

\section{Acknowledgments}

Parts of this work would not have been possible without the assistance of DIMES (Delft Institute of Microsystems and Nanoelectronics).

\section{References}

[1] Kim C, Knoemschild C, Liu B and Kim J 2007 J. Sel. Top. Quantum Electron. 13 322-9

[2] Christian R G 1966 The theory of oscillating-vane vacuum gauges Vacuum 16175

[3] Newel W E 1967 Miniaturization of tuning forks Science $1611320-6$

[4] Kadar Z, Kindt W, Bossche A and Mollinger J 1996 Quality factor of torsional resonators in the low-pressure region Sensors Actuators A 53 299-303

[5] Li B, Wu H, Zhu C and Liu J 1999 The theoretical analysis on damping characteristics of resonant microbeam in vacuum Sensors Actuators A 77 191-4

[6] Bao M, Yang H, Yin H and Sun Y 2002 Energy transfer model for squeeze-film air damping in low vacuum J. Micromech. Microeng. 12 341-6

[7] Sumali H 2007 Squeeze-film damping in the free molecular regime: model validation and measurement on a MEMS $J$. Micromech. Microeng. 17 2231-40

[8] Singer N C and Seering W P 1996 J. Dyn. Syst. Meas. Control Trans. ASME 112 76-8

[9] Miller K, Cowen A, Hames G and Hardy B 2004 Soimumps design handbook, from http://www.memsrus.com/docu ments/SOIMUMPs.dr.v4.pdf, 2004 